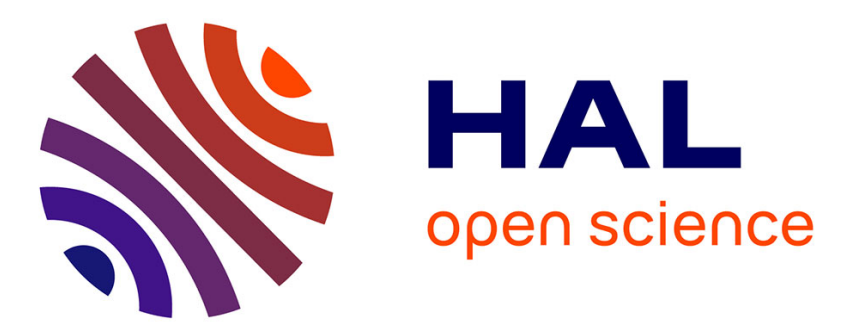

\title{
A simple fully well-balanced and entropy preserving scheme for the shallow-water equations
}

Christophe Berthon, Victor Michel-Dansac

\section{To cite this version:}

Christophe Berthon, Victor Michel-Dansac. A simple fully well-balanced and entropy preserving scheme for the shallow-water equations. Applied Mathematics Letters, 2018, 86, pp.284-290. 10.1016/j.aml.2018.07.013 . hal-01708991v2

\section{HAL Id: hal-01708991 https://hal.science/hal-01708991v2}

Submitted on 9 Jul 2018

HAL is a multi-disciplinary open access archive for the deposit and dissemination of scientific research documents, whether they are published or not. The documents may come from teaching and research institutions in France or abroad, or from public or private research centers.
L'archive ouverte pluridisciplinaire HAL, est destinée au dépôt et à la diffusion de documents scientifiques de niveau recherche, publiés ou non, émanant des établissements d'enseignement et de recherche français ou étrangers, des laboratoires publics ou privés. 


\title{
A simple fully well-balanced and entropy preserving scheme for the shallow-water equations
}

\author{
Christophe Berthon ${ }^{\mathrm{a}}$, Victor Michel-Dansac ${ }^{\mathrm{b}, *}$ \\ ${ }^{a}$ Laboratoire de Mathématiques Jean Leray, Université de Nantes, 2 rue de la Houssinière, 44322 Nantes Cedex 3 , France \\ ${ }^{b}$ Institut de Mathématiques de Toulouse, INSA Toulouse et Université Toulouse 3 Paul Sabatier, 135 avenue de Rangueil, \\ 31077 Toulouse Cedex 4, France
}

\begin{abstract}
In this communication, we consider a numerical scheme for the shallow-water system. The scheme under consideration has been proven to preserve the positivity of the water height and to be fully well-balanced, i.e. to exactly preserve the smooth moving steady state solutions of the shallow-water equations with the topography source term. The goal of this work is to prove a discrete entropy inequality satisfied by this scheme.
\end{abstract}

Keywords: shallow-water equations, Godunov-type schemes, well-balanced schemes, moving steady states, entropy-satisfying schemes $2000 \mathrm{MSC}: 65 \mathrm{M} 08,65 \mathrm{M} 12$

\section{Introduction}

In this work, we consider the shallow-water approximation of free-surface flows in a longitudinal channel. In one space dimension, this model is governed by the following system:

$$
\left\{\begin{array}{l}
\partial_{t} h+\partial_{x} q=0 \\
\partial_{t} q+\partial_{x}\left(\frac{q^{2}}{h}+\frac{1}{2} g h^{2}\right)=-g h \partial_{x} Z
\end{array}\right.
$$

where $h(x, t)$ is the water height, $q(x, t)$ is its discharge (equal to $h u$, where $u$ is the water velocity), $g$ is the gravity constant and $Z$ is the smooth given bottom topography. In order to shorten the notations, we rewrite (1) under the classical form of a conservation law with a source term $\partial_{t} W+\partial_{x} F(W)=S(W)$, where we have set:

$$
W=\left(\begin{array}{l}
h \\
q
\end{array}\right), \quad F(W)=\left(\begin{array}{c}
q \\
\frac{q^{2}}{h}+\frac{1}{2} g h^{2}
\end{array}\right), \quad S(W)=\left(\begin{array}{c}
0 \\
-g h \partial_{x} Z
\end{array}\right) .
$$

In the present work, we assume that the flow is always far from dry areas. The conserved variables $W$ thus lie in the set of admissible states $\Omega$, which prescribes a physically admissible positive water height:

$$
\Omega=\left\{W={ }^{t}(h, q) \in \mathbb{R}^{2} \mid h>0\right\} .
$$

In addition, note that the following natural entropy inequality, satisfied by the admissible entropy weak solutions, arises from this system:

$$
\partial_{t} \eta(W)+\partial_{x} G(W) \leq-g q \partial_{x} Z
$$

\footnotetext{
*Corresponding author

Email addresses: christophe.berthon@univ-nantes.fr (Christophe Berthon), vmd@math.univ-toulouse.fr (Victor Michel-Dansac )
}

Preprint submitted to Applied Mathematical Letters 
where the entropy $\eta$ and the entropy flux $G$ are defined by:

$$
\eta(W)=\frac{1}{2} \frac{q^{2}}{h}+\frac{1}{2} g h^{2} \quad \text { and } \quad G(W)=\frac{q}{h}\left(\frac{1}{2} \frac{q^{2}}{h}+g h^{2}\right) .
$$

Note that, according to [5], the non-conservative entropy inequality (2) can be recast under the following equivalent conservative form:

$$
\partial_{t}(\eta(W)+g h Z)+\partial_{x}(G(W)+g q Z) \leq 0 .
$$

Preserving the steady state solutions of the shallow-water equations, i.e. solutions of (1) such that $\partial_{t} W=0$, has been a major challenge of the last two decades. The steady states at rest, describing motionless water over a possibly complex bottom topography, are obtained by assuming $q=0$ as well as $\partial_{t} W=0$, to get $\partial_{x}(h+Z)=0$. These steady states have been the focus of much work, and several relevant numerical schemes have been developed (see for instance [1,2,4], but this list is far from being exhaustive). Such steady solutions are widely encountered in real-world applications, and being able to preserve them is crucial for a numerical method. Conversely, less work has been undertaken on so-called fully well-balanced schemes, which exactly preserve the smooth moving steady state solutions, defined by:

$$
\left\{\begin{array}{l}
q=\mathrm{cst}, \\
\frac{q^{2}}{2 h^{2}}+g(h+Z)=\mathrm{cst} .
\end{array}\right.
$$

In particular, few first-order schemes have been developed (see for instance $[8,3,10]$ ). Nice properties for a scheme to possess are, in addition to being well-balanced, the preservation of the admissible set $\Omega$ (i.e. the preservation of the water height positivity, also called the robustness property) and a discrete analogue to the entropy inequality (2).

In [3], the authors derive a scheme with the three previous properties. However, in practice, this scheme is computationally too costly, since it involves finding the roots of a fifth-order polynomial. The scheme proposed in [10] corrects this cost shortcoming by introducing an approach taking into account a generic source term, leading to a suitable linearization. However, an entropy inequality was not exhibited. The goal of the present work is to establish an entropy inequality satisfied, in some sense to be prescribed, by the numerical scheme proposed in [10].

\section{Presentation of the numerical scheme}

In this Section, for the sake of completeness, we give the numerical scheme developed in [10]. It falls within the framework of finite volume schemes, and more specifically of Godunov-type schemes (see [9] for instance). As usual, we introduce a discretization of the one-dimensional space domain $\mathbb{R}$ by defining cells of constant volume $\Delta x$. The cell $c_{i}=\left(x_{i-1 / 2}, x_{i+1 / 2}\right)$ has center located at $x_{i}$, with $x_{i \pm 1 / 2}=x_{i} \pm \Delta x / 2$. The time step is denoted by $\Delta t$ and it is restricted according to the following CFL-like condition:

$$
\Delta t \leq \frac{\Delta x}{2 \Lambda}, \quad \text { with } \Lambda=\max _{i \in \mathbb{Z}}\left(-\lambda_{i+1 / 2}^{L}, \lambda_{i+1 / 2}^{R}\right),
$$

where $\lambda_{i+1 / 2}^{L}$ and $\lambda_{i+1 / 2}^{R}$ are approximations of the characteristic velocities $u \pm \sqrt{g h}$ of the hyperbolic system, to be defined. As prescribed by the finite volume framework, the solution of the shallow-water system (1) is approximated, at time $t^{n}$, by the following function, piecewise constant in each cell $c_{i}$ :

$$
W^{\Delta}\left(x, t^{n}\right)=W_{i}^{n} \text { if } x \in\left(x_{i-1 / 2}, x_{i+1 / 2}\right) .
$$

Godunov's scheme is based on the exact solution of the Riemann problems arising between the piecewise constant approximations at the interfaces of two consecutive cells. Using exact solutions, however, is inadvisable in practice. Indeed, source terms and nonlinearities prevent the derivation of analytical exact solutions 
to Riemann problems. Thus, we turn to a Godunov-type scheme, where a consistent approximation of the exact solution are introduced. The updated solution, at time $t^{n}+\Delta t$ and within the cell $c_{i}$, is then given by:

$$
W_{i}^{n+1}:=\frac{1}{\Delta x} \int_{x_{i-1 / 2}}^{x_{i+1 / 2}} \widetilde{W}\left(x, t^{n}+\Delta t\right) d x
$$

where we have set

$$
\widetilde{W}\left(x, t^{n}+t\right)=\widetilde{W}_{\mathcal{R}}\left(\frac{x-x_{i+1 / 2}}{t} ; W_{i}^{n}, W_{i+1}^{n}\right) \text { for } x \in\left(x_{i}, x_{i+1}\right),
$$

with $\widetilde{W}_{\mathcal{R}}\left(x / t ; W_{L}, W_{R}\right)$ a relevant approximation of the solution to the Riemann problem for (1) between arbitrary states $W_{L} \in \Omega$ and $W_{R} \in \Omega$. As a consequence, the updated approximate solution at time $t^{n+1}$ is also piecewise constant on each cell, and it is given by

$$
W^{\Delta}\left(x, t^{n+1}\right)=W_{i}^{n+1} \text { if } x \in\left(x_{i-1 / 2}, x_{i+1 / 2}\right) .
$$

In [10], the authors prescribe the following two-state approximate Riemann solver in order to recover necessary properties of consistency and well-balance:

$$
\widetilde{W}_{\mathcal{R}}\left(\frac{x}{t} ; W_{L}, W_{R}\right)= \begin{cases}W_{L} & \text { if } x / t<\lambda_{L}\left(W_{L}, W_{R}\right), \\ W_{L}^{*}\left(W_{L}, W_{R}\right) & \text { if } \lambda_{L}\left(W_{L}, W_{R}\right)<x / t<0, \\ W_{R}^{*}\left(W_{L}, W_{R}\right) & \text { if } 0<x / t<\lambda_{R}\left(W_{L}, W_{R}\right), \\ W_{R} & \text { if } x / t>\lambda_{R}\left(W_{L}, W_{R}\right) .\end{cases}
$$

Note the presence of a stationary wave, of velocity 0 , separating the states $W_{L}^{*}$ and $W_{R}^{*}$. This wave corresponds to the action of the source term. We then get the following relation by computing the integral in (6):

$$
W_{i}^{n+1}=W_{i}^{n}-\frac{\Delta t}{\Delta x}\left[\lambda_{i+1 / 2}^{L}\left(W_{i+1 / 2}^{L, *}-W_{i}^{n}\right)-\lambda_{i-1 / 2}^{R}\left(W_{i-1 / 2}^{R, *}-W_{i}^{n}\right)\right],
$$

where we have set:

$$
\begin{array}{cc}
\lambda_{i+1 / 2}^{L}=\lambda_{L}\left(W_{i}^{n}, W_{i+1}^{n}\right), & \lambda_{i-1 / 2}^{R}=\lambda_{R}\left(W_{i-1}^{n}, W_{i}^{n}\right), \\
W_{i+1 / 2}^{L, *}=W_{L}^{*}\left(W_{i}^{n}, W_{i+1}^{n}\right), & W_{i-1 / 2}^{R, *}=W_{R}^{*}\left(W_{i-1}^{n}, W_{i}^{n}\right) .
\end{array}
$$

In (7), the velocities $\lambda_{L}\left(W_{L}, W_{R}\right)$ and $\lambda_{R}\left(W_{L}, W_{R}\right)$ are approximations of the characteristic velocities $u \pm \sqrt{g h}$ of the hyperbolic system. For instance, these approximations can be defined as follows:

$$
\lambda_{L}=\min \left(-\left|u_{L}\right|-\sqrt{g h_{L}},-\left|u_{R}\right|-\sqrt{g h_{R}},-\varepsilon_{\lambda}\right), \quad \lambda_{R}=\max \left(\left|u_{L}\right|+\sqrt{g h_{L}},\left|u_{R}\right|+\sqrt{g h_{R}}, \varepsilon_{\lambda}\right),
$$

where we set $\varepsilon_{\lambda}=10^{-10}$ to ensure that $\lambda_{L}<0<\lambda_{R}$. Let us underline that finer choices, where $\lambda_{L} \neq-\lambda_{R}$, can be adopted (for instance see [12]). The intermediate states are $W_{L}^{*}\left(W_{L}, W_{R}\right)={ }^{t}\left(h_{L}^{*}, q^{*}\right)$ and $W_{R}^{*}\left(W_{L}, W_{R}\right)={ }^{t}\left(h_{R}^{*}, q^{*}\right)$, where the intermediate heights and discharge are given by:

$$
q^{*}=q_{H L L}+\frac{\bar{S} \Delta x}{\lambda_{R}-\lambda_{L}}, \quad h_{L}^{*}=h_{H L L}-\frac{\lambda_{R} \bar{S} \Delta x}{\alpha\left(\lambda_{R}-\lambda_{L}\right)}, \quad h_{R}^{*}=h_{H L L}-\frac{\lambda_{L} \bar{S} \Delta x}{\alpha\left(\lambda_{R}-\lambda_{L}\right)} .
$$

In (9), we have introduced the intermediate state of the HLL solver (see [9]), defined as follows:

$$
W_{H L L}=\left(\begin{array}{c}
h_{H L L} \\
q_{H L L}
\end{array}\right)=\frac{\lambda_{R}}{\lambda_{R}-\lambda_{L}} W_{R}-\frac{\lambda_{L}}{\lambda_{R}-\lambda_{L}} W_{L}-\frac{1}{\lambda_{R}-\lambda_{L}}\left(F\left(W_{R}\right)-F\left(W_{L}\right)\right) .
$$

Moreover, the quantity $\alpha$ is given by $\alpha=-\left(q^{*}\right)^{2} /\left(h_{L} h_{R}\right)+g\left(h_{L}+h_{R}\right) / 2$. Finally, the quantity $\bar{S}$ is a consistent approximation of the source term $-g h \partial_{x} Z$, given by:

$$
\bar{S} \Delta x=-g \frac{2 h_{L} h_{R}}{h_{L}+h_{R}}\left(Z_{R}-Z_{L}\right)+\frac{g}{2} \frac{[h]_{c}^{3}}{h_{L}+h_{R}},
$$


where $[h]_{c}$ is a cutoff of $[h]=h_{R}-h_{L}$, defined with a positive constant $C$ that does not depend on $\Delta x$ :

$$
[h]_{c}= \begin{cases}h_{R}-h_{L} & \text { if }\left|h_{R}-h_{L}\right| \leq C \Delta x \\ \operatorname{sign}\left(h_{R}-h_{L}\right) C \Delta x & \text { otherwise }\end{cases}
$$

After [10], this cutoff turns out to be essential to ensure the required consistency of $\bar{S}$ with the continuous source term.

The numerical scheme from [10] is thus recalled. Let us finish by remarking that the time update (8) can be rewritten under the usual flux-source formulation:

$$
W_{i}^{n+1}=W_{i}^{n}-\frac{\Delta t}{\Delta x}\left(\mathcal{F}_{i+1 / 2}^{n}-\mathcal{F}_{i-1 / 2}^{n}\right)+\frac{\Delta t}{2}\left(\mathcal{S}_{i+1 / 2}^{n}+\mathcal{S}_{i-1 / 2}^{n}\right),
$$

where the expressions of the numerical flux function $\mathcal{F}$ and the numerical source term $\mathcal{S}$ are explicitly given in [10]. Note that the numerical scheme is in conservative form if the topography is flat, i.e. if $\partial_{x} Z=0$.

We finally state the following result, proven in [10].

Theorem 2.1 ([10]). Assume that $W_{i}^{n} \in \Omega$ for all $i \in \mathbb{Z}$. Then, under the CFL-like condition (5), and for a small enough $\Delta x$, the scheme (12) is:

- consistent with the shallow-water system (1);

- robust: for all $i \in \mathbb{Z}$, if $W_{i}^{n} \in \Omega$, then $W_{i}^{n+1} \in \Omega$;

- fully well-balanced: if the flow is a steady state according to (4), i.e. if there exists two constants $q_{0}$ and $\Phi_{0}$ such that, for all $i \in \mathbb{Z}, q_{i}^{n}=q_{0}$ and $\frac{\left(q_{i}^{n}\right)^{2}}{2\left(h_{i}^{n}\right)^{2}}+g\left(h_{i}^{n}+Z_{i}\right)=\Phi_{0}$, then, for all $i \in \mathbb{Z}, W_{i}^{n+1}=W_{i}^{n}$.

\section{An entropy inequality}

According to [9], a Godunov-type scheme applied to the homogeneous system (i.e. with $\partial_{x} Z=0$ ) is entropy-satisfying if the following inequality is satisfied under the CFL condition (5):

$$
\frac{1}{\Delta x} \int_{-\Delta x / 2}^{\Delta x / 2} \eta\left(\widetilde{W}_{\mathcal{R}}\left(\frac{x}{\Delta t} ; W_{L}, W_{R}\right)\right) d x \leq \frac{1}{2}\left(\eta\left(W_{L}\right)+\eta\left(W_{R}\right)\right)-\frac{\Delta t}{\Delta x}\left(G\left(W_{R}\right)-G\left(W_{L}\right)\right),
$$

where $\widetilde{W}_{\mathcal{R}}$ is the approximate Riemann solution (7). As soon as the topography is non-flat, the above formula contains a new term, denoted by $\mathcal{T}\left(W_{L}, W_{R}\right)$ and consistent with the source term $-g q \partial_{x} Z$ in (2). In addition, let us underline that, in order to correctly prove the well-known Lax-Wendroff Theorem (see our main result, Theorem 3.3), the estimation (13) must be divided by $\Delta x$. As a consequence, we immediately note that (13) can relax up to $\mathcal{O}\left(\Delta x^{1+\delta}\right)$, with $\delta>0$ (see for instance [6,3]). In the present work, we will obtain a relaxed estimation with $\delta=1$. Also, according to the two-state definition of $\widetilde{W}_{\mathcal{R}}$, the integral of the left-hand side of (13) can be computed explicitly and recast with respect to $\eta_{L}^{*}:=\eta\left(W_{L}^{*}\right)$ and $\eta_{R}^{*}:=\eta\left(W_{R}^{*}\right)$, as follows:

$$
\lambda_{R} \eta_{R}^{*}-\lambda_{L} \eta_{L}^{*} \leq \lambda_{R} \eta_{R}-\lambda_{L} \eta_{L}-\left(G_{R}-G_{L}\right)+\mathcal{T}\left(W_{L}, W_{R}\right) \Delta x+\mathcal{O}\left(\Delta x^{2}\right),
$$

where, with clear notations, we have set $\eta_{L}:=\eta\left(W_{L}\right), G_{L}:=G\left(W_{L}\right)$, and so on. After [9], let us underline the following inequality:

$$
\left(\lambda_{R}-\lambda_{L}\right) \eta_{H L L} \leq \lambda_{R} \eta_{R}-\lambda_{L} \eta_{L}-\left(G_{R}-G_{L}\right),
$$

where we have set $\eta_{H L L}=\eta\left(W_{H L L}\right)$, with $W_{H L L}$ defined by (10). Therefore, (14) holds as soon as the following estimation is established:

$$
\lambda_{R} \eta_{R}^{*}-\lambda_{L} \eta_{L}^{*}=\left(\lambda_{R}-\lambda_{L}\right) \eta_{H L L}+\mathcal{T}\left(W_{L}, W_{R}\right) \Delta x+\mathcal{O}\left(\Delta x^{2}\right) .
$$


Lemma 3.1. For a smooth topography function, the following estimation holds:

$$
\lambda_{R} \eta_{R}^{*}-\lambda_{L} \eta_{L}^{*}=\left(\lambda_{R}-\lambda_{L}\right) \eta_{H L L}+\mathcal{T}\left(W_{L}, W_{R}\right) \Delta x+\mathcal{O}\left(\Delta x^{2}\right)
$$

where the term $\mathcal{T}\left(W_{L}, W_{R}\right)$ is consistent with the topography source term $-g q \partial_{x} Z$, and it is given by:

$$
\mathcal{T}\left(W_{L}, W_{R}\right)=-g \frac{2 h_{L} h_{R}}{h_{L}+h_{R}} \frac{q_{H L L}}{h_{H L L}} \frac{Z_{R}-Z_{L}}{\Delta x} .
$$

Proof: By definition (2) of the entropy function $\eta$, we immediately have

$$
\lambda_{R} \eta_{R}^{*}-\lambda_{L} \eta_{L}^{*}=\frac{1}{2}\left(\lambda_{R} \frac{\left(q^{*}\right)^{2}}{h_{R}^{*}}-\lambda_{L} \frac{\left(q^{*}\right)^{2}}{h_{L}^{*}}\right)+\frac{g}{2}\left(\lambda_{R}\left(h_{R}^{*}\right)^{2}-\lambda_{L}\left(h_{L}^{*}\right)^{2}\right) .
$$

The core of this proof lies in a relevant expansion with respect to $\Delta x$ of the intermediate states $h_{L}^{*}, h_{R}^{*}$ and $q^{*}$ given by (9). Indeed, since the topography function is assumed to be smooth, we get $Z_{R}-Z_{L}=\mathcal{O}(\Delta x)$. As a consequence, the definition (11) of the approximate source term yields the following relation:

$$
\bar{S} \Delta x=-g \frac{2 h_{L} h_{R}}{h_{L}+h_{R}}\left(Z_{R}-Z_{L}\right)+\mathcal{O}\left(\Delta x^{3}\right) .
$$

Equipped with such an expansion, the intermediate states turn out to be a perturbation of the HLL intermediate state (10). Indeed, plugging (17) into (9), we have the following estimations:

$q^{*}=q_{H L L}-\frac{g \tilde{h} \Delta Z}{\lambda_{R}-\lambda_{L}}+\mathcal{O}\left(\Delta x^{3}\right), h_{L}^{*}=h_{H L L}+\frac{g \tilde{h} \Delta Z}{\alpha} \frac{\lambda_{R}}{\lambda_{R}-\lambda_{L}}+\mathcal{O}\left(\Delta x^{3}\right), h_{R}^{*}=h_{H L L}+\frac{g \tilde{h} \Delta Z}{\alpha} \frac{\lambda_{L}}{\lambda_{R}-\lambda_{L}}+\mathcal{O}\left(\Delta x^{3}\right)$,

where we have set $\Delta Z=Z_{R}-Z_{L}$ and $\tilde{h}=\frac{2 h_{L} h_{R}}{h_{L}+h_{R}}$. As a consequence, we obtain the following relations:

$$
\begin{aligned}
& \left(q^{*}\right)^{2}=q_{H L L}^{2}-2 q_{H L L} \frac{g \tilde{h} \Delta Z}{\lambda_{R}-\lambda_{L}}+\mathcal{O}\left(\Delta x^{2}\right), \\
& \frac{1}{h_{L}^{*}}=\frac{1}{h_{H L L}}-\frac{1}{h_{H L L}^{2}} \frac{g \tilde{h} \Delta Z}{\alpha} \frac{\lambda_{R}}{\lambda_{R}-\lambda_{L}}+\mathcal{O}\left(\Delta x^{2}\right), \quad\left(h_{L}^{*}\right)^{2}=h_{H L L}^{2}+2 h_{H L L} \frac{g \tilde{h} \Delta Z}{\alpha} \frac{\lambda_{R}}{\lambda_{R}-\lambda_{L}}+\mathcal{O}\left(\Delta x^{2}\right), \\
& \frac{1}{h_{R}^{*}}=\frac{1}{h_{H L L}}-\frac{1}{h_{H L L}^{2}} \frac{g \tilde{h} \Delta Z}{\alpha} \frac{\lambda_{L}}{\lambda_{R}-\lambda_{L}}+\mathcal{O}\left(\Delta x^{2}\right), \quad\left(h_{R}^{*}\right)^{2}=h_{H L L}^{2}+2 h_{H L L} \frac{g \tilde{h} \Delta Z}{\alpha} \frac{\lambda_{L}}{\lambda_{R}-\lambda_{L}}+\mathcal{O}\left(\Delta x^{2}\right) .
\end{aligned}
$$

Arguing the above relations and performing straightforward computations yields the following estimations:

$$
\begin{aligned}
& \lambda_{R} \frac{\left(q^{*}\right)^{2}}{h_{R}^{*}}-\lambda_{L} \frac{\left(q^{*}\right)^{2}}{h_{L}^{*}}=\left(\lambda_{R}-\lambda_{L}\right) \frac{q_{H L L}^{2}}{h_{H L L}}-2 \frac{q_{H L L}}{h_{H L L}} g \tilde{h} \Delta Z+\mathcal{O}\left(\Delta x^{2}\right), \\
& \lambda_{R}\left(h_{R}^{*}\right)^{2}-\lambda_{L}\left(h_{L}^{*}\right)^{2}=\left(\lambda_{R}-\lambda_{L}\right) h_{H L L}^{2}+\mathcal{O}\left(\Delta x^{2}\right) .
\end{aligned}
$$

Combining these estimations into (16), we immediately get:

$$
\lambda_{R} \eta_{R}^{*}-\lambda_{L} \eta_{L}^{*}=\left(\lambda_{R}-\lambda_{L}\right)\left(\frac{1}{2} \frac{q_{H L L}^{2}}{h_{H L L}}+\frac{g}{2} h_{H L L}^{2}\right)-g \tilde{h} \frac{q_{H L L}}{h_{H L L}} \Delta Z+\mathcal{O}\left(\Delta x^{2}\right),
$$

which is nothing but the expected estimation (15). The proof is thus achieved.

We now state the discrete entropy inequality satisfied by the numerical scheme.

Theorem 3.2. The numerical scheme (12) satisfies the following discrete entropy inequality:

$$
\eta_{i}^{n+1} \leq \eta_{i}^{n}-\frac{\Delta t}{\Delta x}\left(\mathcal{G}\left(W_{i}^{n}, W_{i+1}^{n}\right)-\mathcal{G}\left(W_{i-1}^{n}, W_{i}^{n}\right)\right)+\Delta t \mathcal{T}_{i-1 / 2}^{n}+\mathcal{O}\left(\Delta x^{2}\right),
$$


where the numerical entropy flux $\mathcal{G}$ is given by:

$$
\mathcal{G}\left(W_{L}, W_{R}\right)=G_{L}+\frac{\Delta x}{2 \Delta t} \eta_{L}-\frac{1}{\Delta t} \int_{0}^{\Delta x / 2} \eta\left(\widetilde{W}\left(\frac{x}{\Delta t} ; W_{L}, W_{R}\right)\right) d x
$$

and where the numerical entropy source term $\mathcal{T}_{i-1 / 2}^{n}$ is defined by $\mathcal{T}_{i-1 / 2}^{n}=\mathcal{T}\left(W_{i-1}^{n}, W_{i}^{n}\right)$, with the function $\mathcal{T}\left(W_{L}, W_{R}\right)$ introduced in Lemma 3.1.

Proof: The proof of this result follows immediately from the application of Jensen's inequality and from Lemma 3.1. For more details, the reader is referred to [11].

We conclude this work by an extension of classical Lax-Wendroff theorem, whose proof can be found in [7] for instance.

Theorem 3.3 (Lax-Wendroff). Let us introduce the notation $W^{\Delta}(x, t)=W_{i}^{n}$ for $x \in\left(x_{i-1 / 2}, x_{i+1 / 2}\right)$ and $t \in\left(t^{n}, t^{n+1}\right)$. According to (12), there exists a consistent numerical flux $\mathcal{F}$ and a consistent approximation $\mathcal{S}$ of the topography source term $-g h \partial_{x} Z$ such that

$$
W_{i}^{n+1}=W_{i}^{n}-\frac{\Delta t}{\Delta x}\left(\mathcal{F}_{i+1 / 2}^{n}-\mathcal{F}_{i-1 / 2}^{n}\right)+\frac{\Delta t}{2}\left(\mathcal{S}_{i+1 / 2}^{n}+\mathcal{S}_{i-1 / 2}^{n}\right) .
$$

In addition, according to Theorem 3.2, there exists a consistent numerical entropy flux $\mathcal{G}$ and a consistent approximation $\mathcal{T}$ of the entropy topography source term $-g q \partial_{x} Z$ such that

$$
\eta_{i}^{n+1} \leq \eta_{i}^{n}-\frac{\Delta t}{\Delta x}\left(\mathcal{G}_{i+1 / 2}^{n}-\mathcal{G}_{i-1 / 2}^{n}\right)+\Delta t \mathcal{T}_{i-1 / 2}^{n}+\mathcal{O}\left(\Delta x^{2}\right) .
$$

Assume that $\Delta x$ tends to 0 while preserving a constant ratio $\Delta t / \Delta x$. If, in addition, $W^{\Delta}$ is valued in some compact set $K \in \Omega$, and the sequence $W^{\Delta}$ converges in $L_{\text {loc }}^{1}\left(\mathbb{R} \times \mathbb{R}_{+} ; \Omega\right)$ towards some $W$, then $W$ is an entropy weak solution of (1).

Note that the converged solution $W$ will immediately satisfy the non-conservative version (2) of the entropy inequality. However, according to [5], the conservative version (3) is equivalent to the non-conservative one, and thus the converged solution will also satisfy this conservative entropy inequality. The numerical scheme under consideration therefore converges towards an entropy weak solution of (1).

Acknowledgments. C. Berthon acknowledges the financial support of the ANR-14-CE25-0001 ACHYLLES. V. Michel-Dansac acknowledges the financial support of the Service d'Hydrographie et d'Océanographie de la Marine (SHOM).

[1] E. Audusse, F. Bouchut, M.-O. Bristeau, R. Klein and B. Perthame. A fast and stable well-balanced scheme with hydrostatic reconstruction for shallow water flows. SIAM J. Sci. Comput., 25(6):2050-2065, 2004.

[2] E. Audusse, C. Chalons and P. Ung. A simple well-balanced and positive numerical scheme for the shallow-water system. Commun. Math. Sci., 13(5):1317-1332, 2015.

[3] C. Berthon and C. Chalons. A fully well-balanced, positive and entropy-satisfying Godunov-type method for the shallowwater equations. Math. Comp., 85(299):1281-1307, 2016.

[4] C. Berthon and F. Foucher. Efficient well-balanced hydrostatic upwind schemes for shallow-water equations. J. Comput. Phys., 231(15):4993-5015, 2012.

[5] F. Bouchut. Nonlinear stability of finite volume methods for hyperbolic conservation laws and well-balanced schemes for sources. Frontiers in Mathematics. Birkhäuser Verlag, Basel, 2004.

[6] G. Gallice. Solveurs simples positifs et entropiques pour les systèmes hyperboliques avec terme source. $C$. $R$. Math. Acad. Sci. Paris, 334(8):713-716, 2002.

[7] E. Godlewski and P.-A. Raviart. Numerical approximation of hyperbolic systems of conservation laws, volume 118 of Applied Mathematical Sciences. Springer-Verlag, New York, 1996.

[8] L. Gosse. A well-balanced flux-vector splitting scheme designed for hyperbolic systems of conservation laws with source terms. Comput. Math. Appl., 39(9-10):135-159, 2000.

[9] A. Harten, P. D. Lax, and B. van Leer. On upstream differencing and Godunov-type schemes for hyperbolic conservation laws. SIAM Rev., 25(1):35-61, 1983.

[10] V. Michel-Dansac, C. Berthon, S. Clain, and F. Foucher. A well-balanced scheme for the shallow-water equations with topography. Comput. Math. Appl., 72(3):568-593, 2016.

[11] E. F. Toro. Riemann solvers and numerical methods for fluid dynamics. A practical introduction. Springer-Verlag, Berlin, third edition, 2009.

[12] J.-P. Vila. Simplified Godunov Schemes for $2 \times 2$ Systems of Conservation Laws. SIAM J. Numer. Anal., 1986, 23, $1173-1192$ 